\title{
RENOVACIÓN LÉXICA Y DICCIONARIO: EL NUEVO MOLINER
}

\author{
Gloria Guerrero Ramos y Manuel Fernando Pérez Lagos \\ Universidad de Málaga \\ guerrero@uma.es /mfperez@uma.es
}

\begin{abstract}
Resumen
Nowadays very few people are those who think that neology is an avoidable wrong. We ale have the ability to create new words, either in a spontaneous or conscious way, and it is undeniable that languages constantly renew their lexical wealth and that, therefore, the inventory of words of a language will never be able to be conceived as a static or closed set.

The dictionary is the specifically designed work to gather up this inventory of words and, furthermore, due to its long tradition, it is today conceived as a cultural institution that acts as a depository of the social memory for the meaning of the lexicon of any given linguistic community. In this occasion, in order to verify the movement of lexical renewal, we shall compare and analyse two editions of a great dictionary, the one from Maria Moliner.
\end{abstract}

\section{Introducción}

Hoy muy pocos son ya los que piensan que la neología es un mal evitable. Al contrario, todos somos conscientes de que la neología es una de las manifestaciones principales de la vitalidad de una lengua que quiera mantenerse viva. Hay una necesidad real de crear palabras tanto generales o comunes como especializadas. Diariamente leemos en la prensa y oímos noticias relacionadas con temas científicos, actividades profesionales y asuntos sociales en las que nos encontramos palabras que nos resultan extrañas porque son nuevas. Se refieren a nuevos conceptos y a nuevas maneras de sentir y de pensar a los que nos está conduciendo la sociedad actual. Este movimiento de renovación del vocabulario obedece, por tanto, a factores externos al sistema lingüístico (Azorín, 2000: 212). Sin ir más lejos podríamos analizar la cantidad de palabras nuevas que nos ha dejado el conflicto de Irak, como la de guerra preventiva tan usual ya entre los hablantes de nuestra comunidad lingüística y de la que hace tan sólo unos meses no sabríamos dar una definición.

Todos tenemos la capacidad de crear palabras nuevas, sea de manera espontánea o consciente, y es indudable, pues, que las lenguas renuevan constantemente su caudal léxico y que, por tanto, el inventario de palabras de una lengua nunca se podrá concebir como un conjunto estático o cerrado.

El diccionario es la obra específicamente diseñada para recoger ese inventario de palabras y, además, por su larga tradición, hoy se concibe como una institución cultural que actúa como depósito de la memoria social sobre el significado del léxico de una comunidad lingüística. Una buena forma de constatar el movimiento de renovación léxica del que ha- 
blábamos ha de ser, pues, comparar y analizar dos ediciones de uno de estos diccionarios, es decir, comprobar su actitud ante la neología y los neologismos.

\section{Sobre la neología}

Será preciso, antes de continuar, aclarar qué entendemos por neología y neologismo. Guerrero Ramos (1997: 9-16) ya se ocupó de las definiciones que de neología y neologismo ofrecen los diccionarios generales más representativos de nuestra lengua así como de las que ofrecen algunos específicos. Allí se destacaba que el término neología no aparecía en la mayoría de los diccionarios y tampoco ha sido incluido ahora en las ediciones más recientes. Se trata de un término que, sin embargo, no se duda en usar con el sentido general, que nosotros seguiremos, de "proceso de formación de nuevas unidades léxicas". Por su parte los neologismos, siguiendo a Bastuji (1974), los concebiremos simplemente como unidades léxicas nuevas, es decir, el producto del proceso de neología:

"la distinction entre néologie et néologisme articule une opposition pertinente entre le PROCÉs et le PRODUIT, selon le trait sémantique [ \pm accompli]. Les néologismes sont des unités lexicales nouvelles; la néologie postule un système, un ensemble de regles et conditions qui contraignent la création, le réperage et lèmploi de ces unités nouvelles" (Bastuji, 1974: 6) $)^{2}$.

Seguiremos también la distinción básica, generalmente admitida, entre neologismos de forma, donde el significado y el significante son nuevos (nueva entrada en un diccionario), y neologismos de sentido, donde sólo el significado es nuevo y se acude a un significante ya existente (nueva acepción en el diccionario).

\section{Neología y diccionario}

Otra cuestión que es preciso tratar es lo que podríamos denominar "sentimiento de neologicidad". Como ha dicho R. Dubuc (1999: 143), "se puede considerar que existe un neologismo mientras no desaparezca, para el conjunto de usuarios a quienes concierne la utilización de dicha palabra, el efecto de asombro causado por la innovación léxica". Se ha escrito mucho sobre qué criterios son los que determinan el carácter neológico de un término. Cuando Rey (1976) se plantea la pregunta que da título a su artículo: "Néologisme: un pseudo-concept?" nos ofrece una definición general de neologismo ("une unité nouvelle, de nature lexicale, dans un code linguistique defini" ${ }^{\text {"3) }}$, y analiza, con ella, una serie de cuestiones que van mucho más allá de la concepción generalizada de neologismo = palabra nueva. Entre estas cuestiones estaba la de si se debe considerar como neologismo toda creación léxica por el simple hecho de suponer una novedad. Aprovechaba el planteamiento para

\footnotetext{
Se trata de la definición que apareciera en su día en el diccionario de lingüística de Dubois et alii (1973).

2 También compartiremos con Bastuji que la neología es un hecho específicamente léxico (1974: 6), aunque no llegaremos a concebir el grupo "neología lexical" como redundante, ya que bien puede servir para disipar toda posible ambigüedad entre quienes el término neologia no es exclusivamente empleado para el léxico.

3 Rey (1976: 4).
} 
defender como neologismo, como nueva creación léxica que forma parte del léxico, aquellas nuevas creaciones que están refrendadas por el uso de la colectividad, y rechazar bajo este concepto aquellas novedades que sólo son fruto de la creación ocasional o individual.

Desde este punto de vista, dado que el diccionario recoge un inventario de voces usado por una comunidad lingüística, es posible hablar de neologismos en un diccionario, ya que éste recogerá también aquellas voces nuevas recientemente puestas en uso por la comunidad. Por otra parte, y esto resulta paradójico, el criterio lexicográfico, esto es, el de la constatación de la no presencia en los diccionarios para determinar el carácter neológico de una unidad léxica ha sido uno de los más empleados ${ }^{4}$. No tiene sentido, desde este punto de vista, hablar de neología en el diccionario; no se puede decir, como suele hacerse, que el repertorio lexicográfico de tal o cual diccionario contiene determinado número de neologismos, pues si forman parte de su nomenclatura ya no son neologismos.

No cabe duda de que cuando un diccionario recoge una nueva creación léxica se está dando el primer paso hacia lo que podríamos llamar su desneologización. Por este motivo las obras lexicográficas pueden ser utilizadas como corpus de exclusión para la determinación de los neologismos que compondrían un repertorio neológico, en cierta medida, en "estado de espera" para ver si inicia o no su proceso desneologizador siendo incorporados al diccionario $0^{5}$. Es cierto, entonces, que resulta paradójico hablar de neología en un diccionario; la verdad es que también resulta paradójico que los requisitos imprescindibles para la consideración de un neologismo como tal sean los mismos que acabarán quitándole su carácter neológico: aceptación del neologismo, su difusión y su afincamiento en la lengua. No obstante, es indiscutible que cuando estamos ante un diccionario de edición muy reciente percibimos ("sentimiento de neologicidad") las voces nuevas que contiene y durante cierto tiempo son sentidas y tratadas como tales. Quiere esto decir que, cuando una nueva unidad léxica es recogida en un diccionario, ésta no pierde su carácter neológico inmediatamente. No es fácil, sin embargo, establecer el parámetro temporal que nos diga no sólo desde cuándo una palabra es neologismo sino hasta cuándo. La única precisión temporal que conocemos es la propuesta por Guilbert, quien en el artículo "neologie" del Grand Larousse de la Langue Francaise, ha señalado que la media de vida de un neologismo es de diez años; después de los diez años está completamente integrado en el léxico, desechado u olvidado.

4 Teresa Cabré nos dice que para determinar el carácter neológico de una unidad léxica se han propuesto distintos parámetros que no son excluyentes:

- la diacronia: una unidad es neológica si ha aparecido en un período reciente;

- la lexicografía: una unidad es neológica si no aparece en los diccionarios;

- la inestabilidad sistemática: una unidad es neológica si presenta signos de inestabilidad formal (morfológi$\cos$, gráficos o fonéticos) o semántica;

- la psicología: una unidad es neológica si los hablantes la perciben como una unidad nueva (Cabré, 1993 445).

Y dice un poco más adelante: "Los neólogos, por lo general, priorizan el parámetro de la lexicografia para determinar la neologicidad de una unidad léxica" (1993: 445).

5 El establecimiento de este corpus de exclusión tampoco es tan simple. Piénsese, por ejemplo, que ningún diccionario contiene todas las palabras, siempre presenta una selección; y piénsese, además, que los diccionarios, según sus características, objetivos, usuarios, etc., serán más o menos propensos a la recogida en su repertorio de voces nuevas. Por todo ello no siempre se podrá decir que determinada unidad léxica es un neologismo porque no aparece en los diccionarios usados como corpus de exclusión. 


\section{El diccionario de María Moliner}

Como decíamos al final de nuestra introducción el diccionario es toda una institución cultural reflejo de la competencia léxica de una comunidad. En realidad es, como dice Mejri (1995: 82) la única institución que tiene poder de decisión en materia de léxico: "Elle tire son autorité du fait qu'elle représente pour les usagers le seul moyen permettant de savoir si un mot existe ou non". En otro lugar (1995: 124), tras destacar el valor lingüístico y social del diccionario, nos hace ver que "il représente pour le linguiste s'occupant de néologie un objet précieux dans ce sens qu'il lui fournit la matière première su laquelle il travaille". Se trata, en definitiva, de un punto de partida ideal para conocer sobre el funcionamiento de la neología en una lengua.

Sin embargo, también tenemos que reconocer que cada diccionario refleja una visión diferente de la neología pues ésta ha de pasar por el filtro que supone las características propias del diccionario, los propósitos editoriales y las actitudes de los lexicógrafos. Por todo ello, el valor y la importancia de la investigación sobre neología que realicemos dependerán también de la obra lexicográfica que elijamos.

El diccionario que hemos elegido, el Diccionario de uso del español de María Moliner ${ }^{6}$, generalmente conocido como el Moliner, ha sido durante mucho tiempo el único diccionario de uso con el que ha contado el español. Se trata de un diccionario reconocido como "distinto" de los demás diccionarios del mercado. Manuel Seco nos lo dice así en la presentación de la nueva edición: "La irrupción del Diccionario de uso en el paisaje lexicográfico español supuso una revolución. Era algo auténticamente nuevo y original"?. Pretende servir tanto para descifrar mensajes como para construirlos y ofrece por ello catálogos de sinónimos y palabras afines al pie de numerosas entradas, informa sobre los mecanismos de construcción y el régimen preposicional adecuados en cada caso, da informaciones sobre el contexto de la comunicación y, especialmente, revisa todas las definiciones tradicionalmente heredadas del diccionario académico ${ }^{8}$ Y es que, entre las características propias que lo han hecho diferente tenemos que destacar su intención permanente de ir siempre un poco más allá de lo ofrecido por la Academia. Con respecto al tema de la neología que nos ocupa la propia autora deja clara su posición en la presentación de su obra:

"En cuanto a neologismos de lenguaje general, teniendo en cuenta que la Academia acaba por dar entrada en el léxico oficial a todos los que, sometidos a la prueba de una espera más o menos larga, a veces excesivamente larga, se acreditan de adiciones estables al habla, ha parecido que no debían excluirse de un diccionario "de uso' los que ya llevan en esa espera un tiempo prudencial" (Moliner-66: XXVI).

También a este respecto nos interesan los comentarios que María Moliner ofrece a continuación de la definición de neologismo:

$6 \quad 1$." edición de 1966-1967, con versión en formato electrónico de 1996 (en adelante Moliner-60); 2." cdición de 1998, con versión en formato electrónico de 2001 (en adelante Moliner-98). Para más información sobre esta obra debe consultarse los estudios editados por Casas Gómez et alii (1998), además de la bibliografía allí reunida (capítulo 12).

7 Seco (1998: XI).

8 Nos dice Seco (1998: XII): "la autora se impuso el trabajo de revisar las definiciones heredadas, una por una, en todas las unidades léxicas, con la mira puesta en la claridad del lenguaje expositivo, en la precisión de los matices y en la evitación de los habituales círculos viciosos". 
"Son, en general, considerados legítimos, sin necesidad de que estén sancionados por la Real Academia, los tecnicismos necesarios para designar conceptos nuevos, así como las designaciones científicas formadas con una raíz culta para atender una nueva necesidad, de acuerdo con las normas generales de la derivación"”.

Son afirmaciones que nos podrían llevar a pensar que para María Moliner sólo estaría justificada la neología especializada ${ }^{10}$. Claro que también podemos pensar que María Moliner sólo exige respaldo para los neologismos científico-técnicos y que, en cambio, los neologismos generales no necesitan ser sancionados por la Academia.

Por otra parte, la nueva edición de este diccionario, el Moliner-98, supone el transcurso de más de treinta años, toda una generación, por lo que es de esperar que la renovación léxica sea importante, máxime si se ha tenido la oportunidad de aprovechar los avances ya realizados por otras obras lexicográficas de su calibre. Piénsese, como nos recuerda Seco en la presentación del diccionario, que en el tiempo transcurrido entre una edición y otra, la Academia ha publicado tres ediciones de su diccionario. Sin embargo, no debemos pensar que estamos ante una obra muy diferente; aunque los cambios formales de la reciente edición sean importantes (orden alfabético para todas las entradas, es decir, sin agrupaciones por familias, categoría gramatical en las diferentes acepciones, un lugar propio para las marcas técnicas y dialectales, un artículo desarrollado para cada voz de las que antes sólo se informaba que era "de significado deducible", etc.), en numerosas ocasiones se insiste en que se ha procurado mantener el espíritu inicial de la obra, como puede verse por ejemplo en los comentarios que acabamos de ver que ofrece sobre neologia y neologismo.

Parece, en definitiva, que las cualidades de este diccionario, de todo el mundo conocidas, y las circunstancias temporales entre una edición y otra, hacen idónea su elección para comprobar el movimiento de renovación léxica sufrido por nuestra lengua en los últimos años.

\section{El análisis}

En primer lugar advertiremos que más que hablar de neologismos hablaremos de innovaciones en la última edición del Moliner. Decimos innovaciones porque nos proponemos hacer un análisis tanto cualitativo como cuantitativo a partir de las nuevas entradas y acepciones que se han añadido en esta segunda edición del diccionario. Hay que distinguir, por tanto, entre nueva entrada y neologismo y considerar, siguiendo a Mejri (1995: 127), que "la nouvelle entrée d'un dictionnaire est une unité que les élaborateurs de l'ouvrage ont jugé bon d'ajouter à l'ancienne nomenclature". En consecuencia, los auténticos neologismos constituyen sólo un subconjunto entre las incorporaciones presentadas por el diccionario, pues muchas de estas incorporaciones pueden ser elecciones propias de los lexicógrafos elaboradores de la obra (que hayan decidido, por ejemplo, aumentar el número de entradas referentes a determinado tema, sea general o especializado, y acudan al acervo léxico ya existente) y no siempre voces recientes que han reclamado por sí solas (han hecho necesaria) su presencia en la nomenclatura del diccionario. 
Para realizar la comparación entre las dos ediciones trabajaremos con la versión en CDRom disponible para cada una de ellas, sólo con la intención de facilitar las búsquedas, ya que estas versiones electrónicas no varían con respecto a la edición impresa.

La segunda versión electrónica del Diccionario de uso del español de María Moliner correspondiente a su segunda edición presenta 82.423 lemas o entradas. La versión electrónica de la edición anterior ofrecía la cifra de 78.185. No debemos pensar por ello que son 4.238 las "novedades" presentadas por la nueva edición, ya que los grandes cambios formales que presenta la reciente edición afectan indudablemente a estos recuentos. El propio diccionario habla de "un crecimiento superior al diez por ciento", pero piénsese, por ejemplo, en que la edición anterior presentaba una entrada independiente para los usos verbales en forma pronominal, mientras que ahora "se incluyen generalmente dentro de la forma no pronominal"; las nombres científicos que formaban parte de la nomenclatura del diccionario han pasado a un apéndice final, etc.

Las grandes diferencias de presentación formal de los contenidos en las dos ediciones del diccionario hacen que las herramientas de consulta y análisis que ofrece la versión electrónica de cada una de ellas sean bastante diferentes. Indudablemente no podemos realizar las mismas búsquedas ni, por supuesto, con la misma exactitud, en la versión de la primera edición, que las que podemos conseguir con el sistema de búsquedas avanzadas, simples y complejas, que ofrece la actual versión.

Por todo ello, dadas las dificultades de comparación por separado de cada uno de los posibles aspectos analizables, debido a la ausencia de una clara etiquetación de estos en la primera edición, hemos preferido realizar una comparación de las dos ediciones entrada por entrada. La cala que hemos utilizado abarca toda la letra $\mathrm{A}$. Al ser una comparación directa y manual hemos querido que sea una cala amplia (casi un $12 \%$ de todo el diccionario) y no dispersa para imposibilitar la selección de cortes a partir de preferencias o intereses particulares de análisis y crítica. Pensamos que la muestra estudiada, 9.776 entradas según la segunda versión electrónica, es más que suficiente para encontrar en ella todos los aspectos que con respecto al tema que nos ocupa presenta todo el diccionario.

No queremos ocuparnos sólo de las cantidades y de la proporcionalidad que representan las diferentes novedades que constatemos, nos interesa más controlar su distribución entre las diferentes "zonas léxicas" que constituyen un diccionario (general, regional, especializado, préstamos, etc.) y comprobar las posibles tendencias que se reflejan.

Distribuimos el material de renovación que hemos reunido en dos grandes bloques: material marcado y material sin marcar. Entendemos por marca, siguiendo a Fajardo (1996: 30-31), aquella información concreta sobre un tipo de restricción o condicionamiento en el uso de la unidad léxica. Haremos con el primer bloque de "innovaciones marcadas" tres grupos: el de novedades con marca diatópica, novedades con marca técnica y novedades con marca diafásica. El segundo gran bloque, de "novedades sin marca", constituye el grupo de auténtica renovación léxica general (sin restricciones). Mientras en el primer bloque nos limitaremos a destacar el movimiento de renovación léxica presentado, en este segundo bloque analizaremos los procedimientos de renovación léxica más empleados: combinación de formas, préstamos, acortamiento, etc. ${ }^{11}$

\footnotetext{
11 El segundo y tercer grupo del primer bloque, junto al segundo bloque serán objeto de una segunda parte, ya que en esta ocasión, por razones de espacio, no podemos abarcar todo el estudio sobre la renovación léxica que se ha producido en el nuevo Moliner.
} 
Si hemos realizado estas agrupaciones es porque estamos muy cerca de planteamientos como los de Rey (1976), quien parece distinguir claramente entre lo que serían neologismos comunes y los neologismos específicos que forman parte de un subsistema regional, social, especializado, etc. Para Rey el neologismo es una unidad del léxico cuya forma significante o su relación significante o significado, caracterizada por un funcionamiento efectivo en un modelo de comunicación determinado, no se ha realizado antes en el estadio inmediatamente anterior del sistema o código de lengua; esta novedad, por tanto, debe ser apreciada con relación al código elegido, ya que "selon le modèle de code choisi, on distinguera donc des néologismes en synchronie large et étroite, des néologismes pour la langue dans son ensemble ou pour un usage déterminé, des néologismes dans un contexte thématique spécialisé (technique et science) ou non spécialisé, etc." (Rey, 1976: 17).

Ya que ningún corpus de exclusión es enteramente válido para la identificación de los neologismos dentro del conjunto de novedades reunidas, según lo que más arriba hemos comentado sobre ellos, nos centraremos sobre todo en mostrar el movimiento general de renovación que, según el Moliner, ha sufrido nuestro léxico a lo largo de treinta años, y sólo destacaremos, quizás para "despertar" ese sentimiento de novedad del usuario del que hablaba A. Rey, aquellas innovaciones recogidas en el Moliner-98 que aún no aparecen en la reciente edición académica de 2001 ( $D R A E-200 I$ ), una edición no sólo aparecida tres años después, sino además sorprendentemente abierta a la recepción de novedades después de su tradicional carácter prescriptivo.

\section{"Novedades" diatópicas}

El conjunto de marcas geográficas que ofrece el Moliner-98, unas más generales y otras más específicas, queda estructurado jerárquicamente en el sistema de búsqueda del CDRom en cuatro grupos generales: España, Hispanoamérica, Filipinas y Marruecos. Las dos últimas marcas aparecen en contadas ocasiones (sólo afectan a 56 entradas en todo el diccionario) y no afectan a la cala de la que nos hemos ocupado. Atendemos, por tanto, a los otros dos grandes grupos.

I) El grupo de España aparece subdividido por comunidades autonómicas y algunas de ellas se subdividen a su vez en provincias e incluso en zonas provinciales. Como el número de novedades que constatamos no es muy amplio (especialmente con respecto al grupo hispanoamericano) hemos agrupado estas por comunidades (sumamos, por tanto, las de marca general de la región con las particulares de alguna o algunas provincias). El gráfico que ofrecemos a continuación muestra los datos generales en cuanto a las ocho regiones en las que hemos encontrado novedades.

La lectura que podemos hacer de este gráfico es la siguiente: con la marca general de Andalucía ( $A n d$.) hemos encontrado, en la letra A, 94 acepciones (correspondientes a 84 entradas). 20 de estas acepciones son nuevas para la segunda edición; de las 26 acepciones (y entradas) existentes con la marca de Asturias (Ast.), sólo 1 es nueva; 6 de las 10 de Canarias (Can.); de las 15 que contamos de Cantabria (Cantb.), sólo 2 son nuevas; de Castilla-León (Áv., Burg., León, Pal., Sal., Seg., Sor., Vall., Zam.) hay en la letra A 89 acepciones (82 entradas) de las cuales sólo 7 son nuevas; de las 5 con marca de La Rioja (Rioj.), sólo 1 es nueva; de las 8 de Navarra ( $N a v$.), sólo 2 son nuevas; y, por último, de las 54 acepciones con alguna marca del País Vasco (P. Vasco, Al., Guip., Vit., Vizc.) sólo 2 son nuevas. Tam- 


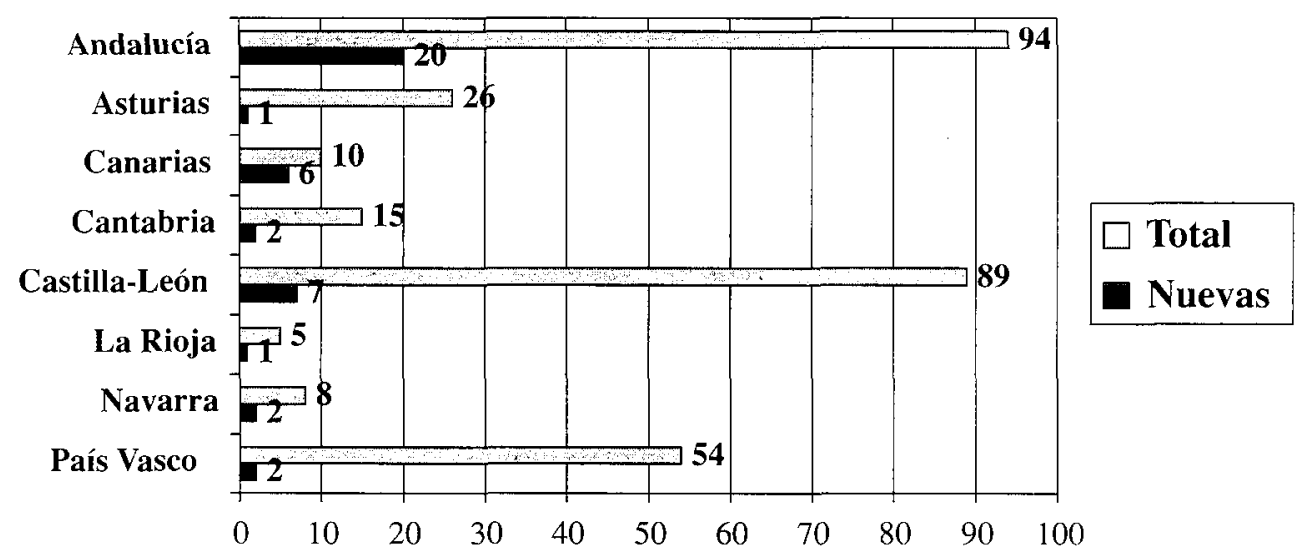

Gráfico 1. Regiones españolas.

bién muestra que en el resto de regiones (Aragón, Castilla-La Mancha, Baleares, Cataluña, Comunidad Valenciana, Extremadura, Galicia, Madrid y Murcia) el diccionario no presenta nuevas acepciones.

En nuestra comparación de este conjunto de nuevas acepciones con el diccionario de la Academia en su edición de 2001, DRAE-2001, encontramos algunas diferencias. He aquí algunas de ellas:

- las acepciones de alambiquero y angurria

alambiquero, -a (And., Hispam.) n. Persona que posee un alambique (destilería) o trabaja en él.

angurria 2 (And., Hispam.) Deseo vehemente.

angurria 4 (And., Hispam.) Hambre insaciable.

no son consideradas de Andalucía;

- las acepciones de achancar de Salamanca y la de alobado de Cantabria

achancar 2 (Sal.) Pisar charcos, barro, etc.

achancar 3 (Sal.) Encajar, encasquetar.

alobado 3 (Cantb.) Alobunado (semejante al lobo, especialmente en el color de pelo).

no aparecen en el diccionario;

- la acepción de abinar de tres provincias de Castilla-León

abinar (Burg., León, Sal.) tr. Binar (arar o cavar por segunda vez las tierras).

aparece sin marca diatópica y con las marcas de registro rural y vulgar;

la acepción de ajotar con marcas, entre otras, de dos provincias de Castilla-León 
ajotar (... León, Sal., Am. C., P. Rico) tr. *Incitar.

aparece también con marca de Extremadura.

Todas estas diferencias que hemos constatado ${ }^{12}$ no se daban en la edición anterior del diccionario académico (DRAE-92). Esto demuestra que el Moliner-98 consultó, para la inclusión de sus novedades, la última edición académica en aquellos momentos, y que las diferencias se deben a la reciente revisión efectuada por la Academia (DRAE-2001).

Tenemos que destacar, no obstante, que la entrada achune de Álava y Navarra

achune (... Ál., Nav.) f. *Ortiga (planta urticácea).

no aparece en el $D R A E-2001$ y tampoco en el $D R A E-92$, y, por tanto, podemos pensar que es la única nueva palabra en este campo presentada por el Moliner-98.

II) El grupo de Hispanoamérica aparece subdividido en cuatro grandes zonas: Méjico, América Central, Antillas y América del Sur. Las tres últimas se presentan a su vez subdivididas en los diferentes países que la forman: América Central (Costa Rica, El Salvador, Guatemala, Honduras, Nicaragua, Panamá), Antillas (Cuba, Puerto Rico y República Dominicana) y América del Sur (Argentina, Bolivia, Chile, Colombia, Ecuador, Perú, Paraguay, Uruguay, Venezuela y la zona de Río de la Plata).

En este apartado hay que diferenciar, por tanto, entre las marcas "generales", referentes a toda la zona hispanoamericana en su conjunto (Hispam.) o a alguno de los tres conjuntos de países distinguidos dentro de ésta (Am. C., Antill. y Am. S.), y las marcas "concretas" de cada uno de los países señalados en las tres últimas, además de la de Méjico (Méj.), que forma grupo independiente.

En el gráfico 2 que ofrecemos a continuación hemos atendido a aquellas acepciones que van etiquetadas con alguna de las marcas "genéricas", sea de toda Hispanoamérica, de alguna de las tres grandes zonas en ella delimitada o de Méjico, ya que este no tiene posteriores subdivisiones.

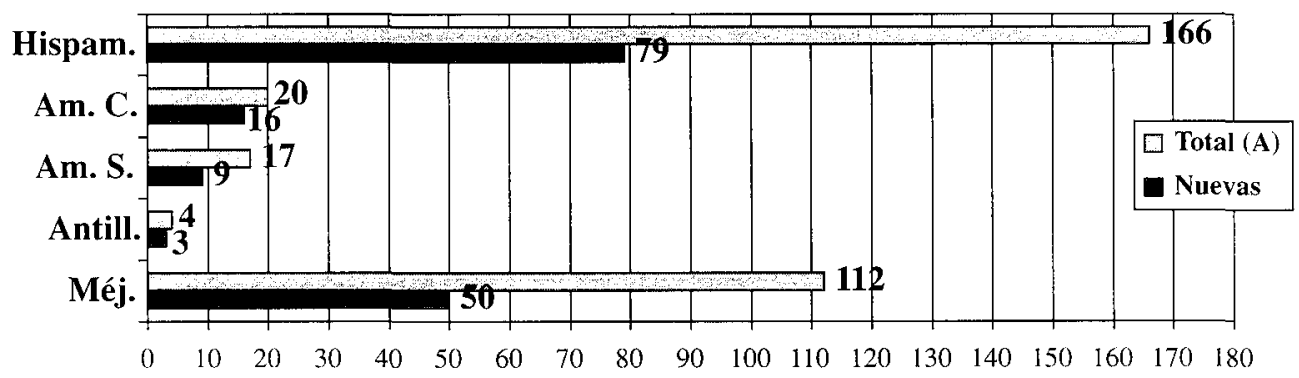

Gráfico 2. Marcas americanas "generales". 
La lectura que se desprende de este gráfico es que de las 166 acepciones (correspondientes a 141 entradas) que encontramos en la letra A del Moliner-98 con la marca referente a toda Hispanoamérica (Hispam.), 79 acepciones han sido incluidas para esta última edición; del mismo modo hemos constatado 20 acepciones (17 entradas) de América Central ( $\mathrm{Am}$. $C$.) de las cuales 16 son nuevas; 17 de América del Sur ( $A m$. S.) de las que 9 son nuevas; 4 de Antillas (Antill.) de las que 3 son nuevas; y, por último, 112 acepciones (en 104 entradas) de Méjico ( $M e ́ j$.$) de las que 50$ no estaban en la edición anterior. Como puede observarse se trata de un número importante de novedades (157) con relación a los totales (319); casi el $50 \%$ de estas acepciones "genéricas" suponen una renovación léxica de la nueva edición.

Haremos a continuación algunos comentarios de este conjunto de "novedades" etiquetadas con alguna de las marcas genéricas mencionadas, especialmente al comprobar qué ha pasado con ellas cinco años más tarde, en el DRAE-2001.

1) La mayoría de las 79 nuevas acepciones que hemos encontrado en el Moliner-98 están, con la marca $A m$. (América) en el $D R A E-2001$. Existen, sin embargo, algunas diferencias.

El afán de claridad del Moliner parece ocasionar que, a veces, presente mayor número de acepciones. Así en ahuesarse, presenta tres acepciones, y en ancheta dos, cuando el DRAE2001 , ofrece dos y una respectivamente.

ahuesarse 1 (Hispam.) prnl. Perder una persona prestigio y estima.

ahuesarse 2 (Hispam.) Perder una cosa su valor, volverse inservible.

ahuesarse 3 (Hispam.) Quedarse sin vender una mercancía.

ancheta 4 (Hispam.) Cosa inoportuna.

ancheta 5 (Hispam.) Cosa que revela descaro o desvergüenza.

Otro grupo de las acepciones no es presentado en el diccionario académico con esta marca general sino con toda una serie de marcas concretas. También ocurría así en la edición académica de 1992, por lo que, al menos en esta ocasión, no se puede decir que el Moliner98 haya seguido literalmente al académico.

ajustar 4 (Hispam.) Contratar a destajo.

amachorrarse (... Hispam.) prnl. Hacerse machorra una hembra.

amanecer 5 (Hispam.) intr. y prnl. Pasar la noche en vela.

amarrar 4 (Hispam.) Vendar o ceñir.

amarrar 6 (Hispam.; muy frec. "amarrársela") Emborracharse.

Tenemos nuevas acepciones como las de abombado, alentado que no aparecen en el $D R A E-2001$ debido a que han sido suprimidas por la Academia de la edición de 1992, edición seguida por el Moliner-98 para estas inclusiones.

abombado', -a 23 (Hispam.) adj. Aturdido, atontado.

alentado (Hispam.) Que ha mejorado o se ha restablecido de una *enfermedad.

Del mismo modo, hemos constatado, como novedad, acepciones tal y como estaban en el $D R A E-92$ y que, ahora, en el $D R A E-2001$, han sido modificadas. Es el caso de agallón.

agallón 4 (Hispam.; pl.) Inflamación de las amígdalas. 
Encontramos una de las acepciones de arrimado que tanto en el DRAE-92 como en el $D R A E-2001$ es presentada como perteneciente a Puerto Rico; quizás todo se deba a que, erróneamente, se haya seguido la marca genérica de la acepción anterior.

arrimado 3 (Hispam.) Persona a la que se concede un pedazo de tierra y que siembra una parte de ella para sí y otra para el dueño de la propiedad.

Tenemos que destacar, finalmente, la acepción hispanoamericana de audífono que no estaba presente en el $D R A E-92$ ni está en el $D R A E-2001$.

audífono 2 (Hispam.; pl.) Auriculares (dispositivo para percibir el sonido de un aparato).

2) Entre las 16 nuevas acepciones recogidas de América Central ( $\mathrm{Am}$. C.) podemos destacar aquellas, como afate y alagartarse, que presentan esta marca genérica cuando el diccionario académico, tanto en la actual edición como en la anterior, ofrece marcas referentes a países concretos.

afate (Am. C., Méj.) m. Ahuate.

alagartarse 2 (Am. C.) Hacerse *tacaño o *avaro.

En el caso de la acepción de amarrar, el DRAE-92 también prefería indicar toda una serie de países concretos, pero ahora en la actual edición se ha convertido en acepción general sin marca dialectal.

amarrar 8 (Am. C., Col., Méj.) prnl. recípr. Casarse.

Destacamos, por último, la acepción de alagartado, no presente en el diccionario académico.

alagartado, -a (Am. C.) Participio adjetivo de "alagartar[se]". *Tacaño, *avaro.

3) Las 9 nuevas acepciones con la marca de América del Sur ( $A m$. C.) están presentes en el diccionario académico, aunque algunas de ellas con ciertas diferencias. Destacaremos sólo acoplar, donde el Moliner ha preferido esta marca genérica cuando el diccionario académico actual muestra, y ya mostraba en la edición anterior, toda una serie de marcas de países concretos.

acoplar 5 (Am. S.) Enganchar uno o varios remolques a un vehículo tractor.

Igual ocurre con sin asco: el DRAE-92 ofrecía en ella las marcas de varios países, pero el Moliner-98 prefirió esta genérica. En esta ocasión el DRAE-2001 sigue la tendencia del Moliner y opta por una marca mucho más genérica aún $(A m$.$) .$

Sin asco (Am. S.). Sin escrúpulos.

4) De las tres nuevas acepciones de Antillas (Antill.) destacaremos sólo el caso de amanezca que aparece tal y como estaba en la edición académica de 1992, pero que ahora el $D R A E-2001$ la ha limitado únicamente a Puerto Rico y República Dominicana. 
amanezca (Méj., Antill.) f. Amanecer (momento de hacerse de día).

5) Entre las 50 nuevas acepciones que hemos recogido con la marca de Méjico (Méj.) observamos que un amplio número de ellas no aparece en la actual edición académica. Las causas principales de estas ausencias son dos:

a) algunas acepciones se muestran tal y como estaba en el $D R A E-92$, pero ahora el DRAE-200: las ha suprimido por completo; es el caso de acalote, alfiler de seguridad, antellevar, archivo

acalote (... Méj.) $\mathrm{m}$. Parte de una laguna o corriente de agua que se limpia de hierbas flotantes para dejar paso a las embarcaciones remeras.

Alfiler de seguridad (Méj.). *Imperdible.

antellevar (Méj.) tr. Atropellar.

archivo 4 (Méj.) Cárcel.

b) otras acepciones se muestran tal y como estaba en el $D R A E-92$, pero ahora la nueva edición académica ha suprimido la marca de Méjico y, en ocasiones, ha añadido otras; es el caso, entre otros, de abatanar, achiquitar, alhajito, arrechar y arruinado.
abatanar 3 (Arg., Bol., Méj.) prnl. Desgastarse o amazacotarse un tejido.
achiquitar (... Col., Guat., Méj., R. Dom.) tr. y prnl. Empequeñecer[se].
alhajito, -a (Ec., Méj.) adj. Bonito, agradable.
arrechar 1 (Am. C., Méj.) intr. Sobrar animación y brío.
arruinado 2 (Am. S., Méj.) Enfermizo, débil.

En el caso de la acepción de acelerado no sigue el Moliner al diccionario académico, ya que no estaba presente en el $D R A E-92$. La nueva edición académica sí la recoge pero la extiende a toda Hispanoamérica $(A m$.).

acelerado 2 (Méj.) Acelerón.

Señalaremos, finalmente, tres de las nuevas acepciones que ni aparecían ni aparecen en el diccionario de la Academia.
¡Ándale! (Méj.). ¡Ándele!
¡Ándele! (Méj.). ¡Venga!
aventar 10 (Méj.) Decidirse.

En el gráfico número 3 que ofrecemos a continuación se presenta cada uno de los países pertenecientes a la zona de América Central (Costa Rica, El Salvador, Guatemala, Honduras, Nicaragua y Panamá) según nos lo muestra el sistema de búsqueda avanzada del Moliner en su actual formato electrónico.

La lectura que se desprende del gráfico es que de las 22 nuevas acepciones ( 21 entradas) de Costa Rica (C. Rica), 15 son nuevas para la actual edición del Moliner; las cuatro acepciones que aparecen con marca de El Salvador (Salv.) han sido incluidas para esta segunda edición; de las 32 acepciones recogidas con marca de Guatemala (Guat.), 21 de ellas son 


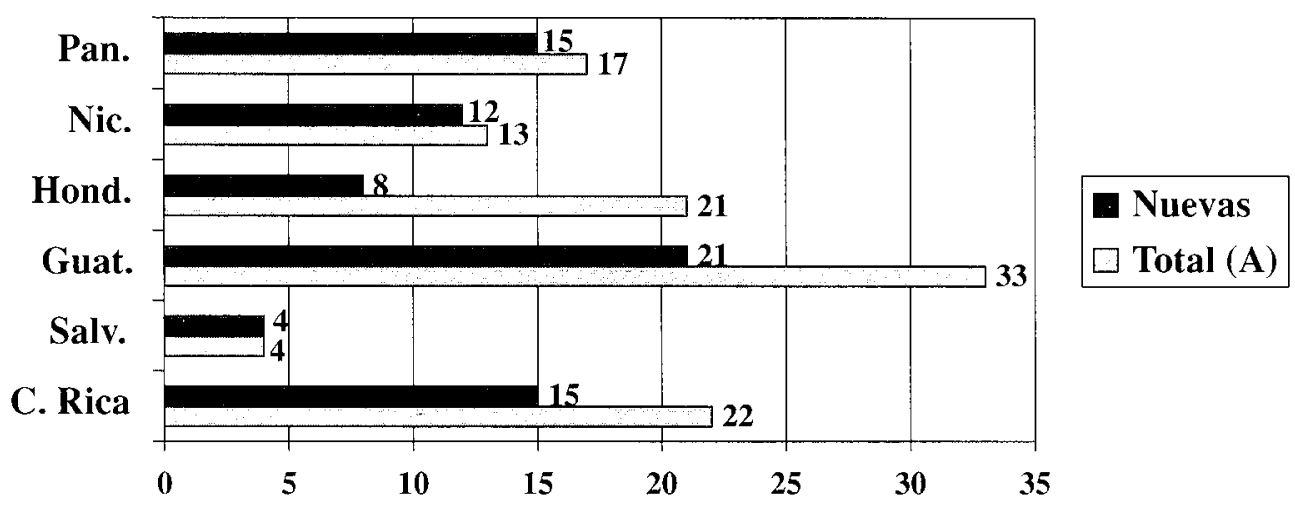

Gráfico 3. Países de América Central.

nuevas; de las 21 con marca de Honduras (Hond.), 8 son nuevas; de las 13 acepciones de Nicaragua (Nic.), 12 son nuevas; $y$, por último, de las 17 acepciones (15 entradas) con la marca de Panamá (Pan.), 15 son nuevas acepciones. A grandes rasgos quiere esto decir que de un total de 110 acepciones recogidas, 75 de ellas son inclusiones de la nueva edición del Moliner, más de un $68 \%$.

Muchas de estas nuevas acepciones aparecen tal y como estaban en la edición académica de 1992, y se diferencian por ello del DRAE-2001, que ha realizado importantes cambios. En algunas de ellas se ha suprimido uno o varios de los países americanos que se ponía (también se añaden, a veces, otros). Así, por ejemplo, para el actual diccionario académico ya no son de Costa Rica abreviar, aguado, andarivel

abreviar (C. Rica, Nic.) prnl. Darse prisa.

aguado 4 (C. Rica, Guat., Méj., Pan., Ven.) adj. *Débil, flojo.

andarivel (Cantb., Col., C. Rica, R. Dom.; pl.) Adornos excesivos, generalmente de mujer:

no son de Guatemala aluzar, amachinarse, amarradijo, apolismar

aluzar (Col., Guat., Méj., P. Rico, R. Dom.) tr. Alumbrar (proyectar luz sobre algo).

amachinarse 2 (Guat., Pan.) prnl. *Abatirse, acobardarse.

amarradijo (Cantb., Col., Guat., Hond.) m. Amarradura, particularmente si está mal hecha.

apolismar 4 (Col., Guat., P. Rico) Quedarse raquitico, no crecer.

no son de Honduras alitranco, andén, amostazar

alitranco (C. Rica, Hond.) m. *Hebilla que tienen los chalecos y pantalones en la parte trasera para ajustarlos.

andén 6 (Col., Guat., Hond.) *Acera de la calle.

amostazar (And., Bol., Col., Ec., Hond., P. Rico) Avergonzarse. 
no son de Panamá apendejarse y atojar

apendejarse (Col., Pan., R. Dom.) prnl. Volverse tonto.

atojar (... C. Rica, Cuba, Pan.) tr. *Azuzar a un perro.

no son de Costa Rica y tampoco de Honduras agualotal, amelcochar

agualotal (C. Rica, Hond., Nic.) m. Aguazal, pantano.

amelcochar 2 (Bol., C. Rica, Ec., Hond., Méj., Par., Perú) Ablandarse, reblandecerse.

Algunas de las nuevas acepciones, marcadas con países de esta zona tal y como estaban en el $D R A E-92$, han sido suprimidas para el $D R A E-2001$; es el caso de apolismar, ajuate, achaparrado, ahuevado

apolismar 2 (C. Rica) prnl. Holgazanear.

ajuate (Salv.) m. Ahuate.

achaparrado 3 (Hond.) Se aplica a la persona apocada.

ahuevado 2 (Col., Nic., Pan., Perú) adj. y n. Acobardado o atontado.

Destacamos también acepciones que no están en el diccionario académico, ni en la actual edición ni en la anterior; lo que muestra que no siempre se sigue a la Academia; es el caso de anaquel, artesa, alfombra, alfombra limpiadora

anaquel 2 (Arg., Ec., Salv., Pan., Perú) Armario de cocina.

artesa 2 (Guat.) Bañera.

alfombra 4 (Cuba, Pan., P. Rico, R. Dom., Ven.) *Felpudo.

alfombra limpiadora (Pan., R. Dom.). *Felpudo.

El gráfico 4 presenta los países que la versión en CD-Rom del nuevo Moliner considera pertenecientes a la zona de América del Sur (Argentina, Bolivia, Chile, Colombia, Ecuador, Perú, Paraguay, Uruguay, Venezuela y zona de Río de la Plata).

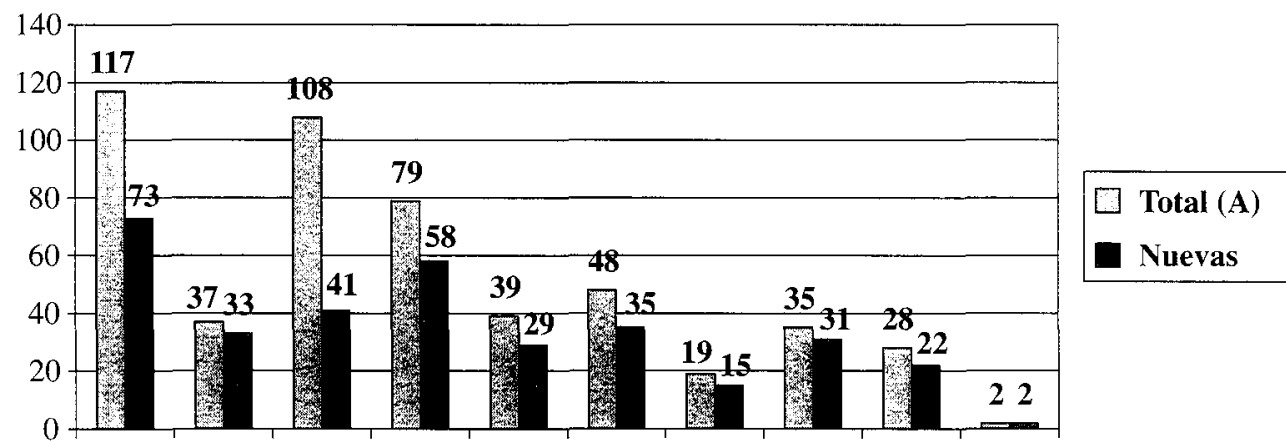

Arg. Bol. Chi. Col. Ec. Perú Par. Ur. Ven. R. PI.

Gráfico 4. Países de América del Sur. 
La primera lectura que se desprende de este gráfico es que de las 117 acepciones (correspondientes a 103 entradas) recogidas con la marca de Argentina (Arg.), 73 son nuevas acepciones incorporadas en esta nueva edición del Moliner; de las 37 acepciones de Bolivia (Bol.), 33 son nuevas; de las 108 acepciones (104 entradas) que recogemos de Chile (Chi.), 41 son nuevas; de las 79 acepciones ( 73 entradas) de Colombia (Col.), 58 son nuevas inclusiones; hay 39 acepciones (36 entradas) con marca de Ecuador (Ec.) y 29 de ellas son nuevas; de las 48 acepciones (45 entradas) de Perú (Perú), 35 son nuevas acepciones; de las 19 acepciones de Paraguay (Par.), hay 15 nuevas; de las 35 acepciones de Uruguay $(U r),$.31 son nuevas; de las 28 acepciones que registramos con marca de Venezuela (Ven.), 22 son acepciones nuevas; y, por último, las dos acepciones recogidas de Río de la Plata $(R$. Pl.) son nuevas inclusiones. Se trata, por tanto, de un aumento léxico superior al $68 \%$, ya que de un total de 496 acepciones, 339 han sido incluidas en el Moliner-98.

En líneas generales casi todas estas nuevas acepciones están presentadas tal y como en su momento aparecieron en el $D R A E-92$, por lo que gran parte de las diferencias que ahora muestran con el actual diccionario académico se debe a los cambios efectuados por el DRAE-2001.

a) En el amplio grupo de Argentina destacaremos aquellas acepciones como afrechero, alagar, aparceria, apepú y apochongarse, incluidas tal y como estaban en el DRAE-92 y que han sido suprimidas por la actual edición académica

afrechero, -a 1 (Arg.) adj. Se aplica al animal que come afrecho.

alagar 2 (Arg., Bol.) prnl. Hacer agua una embarcación.

apepú (... Arg., Par.; Citrus aurantium) m. Naranjo de tronco liso y oscuro, hoja lanceolada de color verde intenso y fruto de sabor amargo y gruesa cáscara llamado del mismo modo.

aparcería 2 (Arg., Ur.) Amistad, compañerismo.

apochongarse (Arg., Ur.) prnl. Asustarse o acobardarse.

Curiosamente, en el caso de afilador, el $D R A E-92$ sólo ofrecía la marca de Ur. (de nuevo el Moliner no sigue fielmente a la Academia), aunque la acepción también ha sido suprimida en el DRAE-2001

afilador 4 (Arg., Ur.; inf.) adj. y n. Se aplica a la persona muy dada a flirtear.

Señalamos también el caso de abarajar, dividido en tres acepciones en el Moliner-98, que estaba en una sola acepción en el $D R A E-92$, y que ahora en el $D R A E-2001$ se ha quedado en la primera acepción y sin la marca de Paraguay

abarajar 1 (Arg., Par., Ur.) tr. Coger una cosa en el aire.

abarajar 2 (Arg., Par., Ur.) Parar un golpe.

abarajar 3 (Arg,, Par., Ur.) Captar la intención con que ha sido dicho o hecho algo.

También hay, como en otras ocasiones, acepciones que ni aparecían ni aparecen en el diccionario académico; es el caso de anaquel (ya visto más arriba), abrochador, amansador, antarquearse, aparador 
abrochador 2 (Arg.) m. o f. Grapadora.

amansador 3 (Arg., Ur.) f. Sala de espera.

antarquearse 1 (Arg; inf.) prnl. Andar muy estirado y con los hombros hacia atrás.

aparador 6 (Arg.) Armario de cocina.

b) Del grupo de nuevas acepciones que llevan marca de Bolivia (Bol.) destacaremos aquellas que seguían fielmente el $D R A E-92$ y ahora han sido suprimidas en la actual edición académica; se trata de abonar, ajear, amojosado y arco

abonar 8 (Bol.) prnl. recípr. Reconciliarse.

ajear ${ }^{2}$ (Bol., Cantb., Ec., Perú) intr. Decir ajos o palabrotas.

amojosado, -a (Bol.) adj. Cubierto de moho.

arco 6 (Bol.) Ceremonia que se celebra en algunas bodas populares para obtener dinero de los invitados.

También hay acepciones que ahora en el $D R A E-2001$ han perdido la marca de Bolivia; es el caso de acatanca, aflar, aisa, amañar y amaño
acatanca (... Arg., Bol.) f. Escarabajo pelotero. Ô Catanga.
afilar 3 (Bol., Ur.) *Prepararse concienzudamente para realizar una tarea.
aisa (... Arg., Bol., Perú) f. Derrumbe en el interior de una mina.
amañar 3 (Arg., Bol., Col., Ec.) prnl. recípr. Amancebarse.
amaño 4 (Arg., Bol., Col., Ec.) Amancebamiento.

Es curioso el caso de achuma, presente en el diccionario de la Academia, tanto en la actual edición como en la anterior, con marca de Argentina y Perú, y que, sin embargo, el Moliner-98 lo incluye como de Bolivia.

achuma (Bol.) f. Planta cactácea de brazos acanalados.

c) Muchas de las nuevas acepciones con marca de Chile tomadas tal y como estaban en el DRAE-92 han sido ahora modificadas por el nuevo diccionario académico. Además de aquellas a las que se les ha añadido nuevas referencias de otros países, hay otras como anteojudo y amarrar que han tomado la marca general hispanoamericana

amarrar 7 (Chi., Nic., Perú, P. Rico) Pactar, concertar.

anteojudo, -a (Arg., Chi., Guat.; desp.) adj. y n. Que usa anteojos.

Acepciones como agauchar, arranchar, amacharse han perdido la marca de Chile en el DRAE-2001

agauchar (Arg., Chi., Par., Ur.) tr. y prnl. Hacer tomar [o adquirir] el porte, los modales o las costumbres de los gauchos.

amacharse 2 (Chi., Méj.) Resistirse, obstinarse.

$\operatorname{arranchar}^{2}$ (de "arrancar"; Chi., Ec., Perú) tr. Quitar una cosa a alguien con violencia.

Dentro del caso anterior habría que incluir jachachay!, en dos acepciones en el Moliner98 pero siguiendo fielmente la acepción del $D R A E-92$, y que ahora el $D R A E-2001$ hace dos 
acepciones pero, suprimiendo casi todas las marcas, la primera queda sólo de Ecuador y la segunda sólo de Colombia.

¡Achachay! 1 (Chi., Col., Ec., Perú) Exclamación con que se expresa sensación de frío o calor.

¡Achachay! 2 (Chi., Col., Ec., Perú) También, exclamación admirativa que equivale a ¡muy bien!

Otras de las nuevas acepciones incluidas tal y como estaban en el DRAE-92 han sido suprimidas para el DRAE-2001; es el caso de al alabado, amuchar y apurón

al alabado (Chi.). Al amanecer:

amuchar 1 (Bol., Chi., R. Pl.) tr. Aumentar el numero o la cantidad de una cosa. apurón 3 (Chi.) Impaciencia.

Seguimos encontrando casos en los que, indudablemente, no se ha seguido a la Academia; la acepción de abrigo que recogemos no aparece ni aparecía en el diccionario académico

abrigo 2 (Chi., Cuba) * Cazadora (prenda de vestir).

d) En el grupo de acepciones de Colombia hay que incluir los comentarios sobre ahuevado y sobre ¡achachay! ya vistos más arriba. La mayoría de las acepciones están como estaban en el DRAE-92 y, por tanto, las diferencias están en las adiciones y modificaciones recientes del $D R A E-2001$. Queremos destacar, no obstante, dos ocasiones en las que no se ha seguido a la Academia; es el caso de amancayo presentado de Colombia mientras el diccionario académico, tanto el de 1992 como el de 2001, lo presenta de América ( $\mathrm{Am}$.) uniendo las formas amancay, amancayo y amancaya (el Moliner-98 los separa y reconoce la forma amancayo de Panamá).

amancayo (Col.; ...) m. Arbusto apocináceo americano de *flores rosadas o blancas y perfumadas; se le atribuye propiedades medicinales.

El otro caso es el de arrochelarse, que en ninguna de sus dos acepciones está presente en el $D R A E-92$ y tampoco en el $D R A E-2001$.

arrochelarse 1 (Col., Ven.) prnl. *Plantarse una caballeria.

arrochelarse 2 (Col., Ven.) *Desbocarse o inquietarse una caballería.

e) En el grupo de acepciones nuevas con marca de Ecuador, además de los casos ya mencionados en los grupos anteriores que también le afectan (jachachay!) queremos destacar aquellas que, estando tomadas del $D R A E-92$, ahora pierden la marca de Ecuador en el $D R A E-2001$; es el caso de aillo y el de cuatro acepciones de acotejar

aíllo (...; Chi., Ec.) m. Cada uno de los grupos en que se divide una comunidad indigena, cuyos miembros suelen ser del mismo linaje.

acotejar 2 (Can., Col., Cuba, Ec., R. Dom.) Colocar objetos ordenadamente.

acotejar 4 (Cuba, Ec.) prnl. Arreglarse con alguien, ponerse de acuerdo sobre cierta cosa. acotejar 6 (Cuba, Ec.) Conseguir un empleo.

acotejar (Can., Cuba, Ec., R. Dom.) Ponerse cómodo. 
Hay una acepción más de acotejar, también tomada del DRAE-92 que, ahora en el $D R A E-2001$, ha sido suprimida

acotejar 5 (Cuba, Ec.) Cohabitar.

También aparecen acepciones en las que no se ha seguido a los diccionarios académicos como en anaquel (ya visto) y en armador

armador 6 (Ec.) Percha (utensilio con un gancho para colgar prendas de vestir).

f) En el grupo de nuevas acepciones de Perú, además de casos ya mencionados en otros grupos que también le afectan (jachachay!, ahuevado, anaquel), señalaremos el caso de almacigado, una acepción tomada tal y como estaba en la edición académica de 1992, que ahora en el nuevo DRAE-2001 ha sido suprimida

almacigado 3 (Perú) Particularmente aplicado al color de la piel, trigueño.

Tampoco encontraremos como marcada de Perú la acepción de apensionarse, que ha sido modificada en el DRAE-2001

apensionarse (Chi., Col., Méj., Perú) prnl. Apenarse.

g) En el grupo de nuevas acepciones que llevan la marca de Paraguay, además de aquellos casos ya mencionados en los grupos ya vistos (abarajar, apepú), tenemos que señalar las que como abatatamiento, agauchado, agauchar, amelcochar, ampalaba o ampalagua y argel, estaban fielmente tomadas del $D R A E-92$, y han perdido la marca de este país en la nueva edición académica

abatatamiento (Arg., Par., Ur.) m. Acción y efecto de abatatar[se].

agauchado, -a (Arg., Chi., Par., Ur.) Participio adjetivo de "agauchar[se]". Parecido a los gauchos o a sus cosas.

agauchar (Arg., Chi., Par., Ur.) tr. y prnl. Hacer tomar [o adquirir] el porte, los modales o las costumbres de los gauchos.

amelcochar 2 (Bol., C. Rica, Ec., Hond., Méj., Par., Perú) Ablandarse, reblandecerse. ampalaba o ampalagua (Arg., Chi., Par., Ur.) f. Nombre de varias serpientes americanas de gran tamaño.

argel 3 (Arg., Par.) Que no tiene gracia ni resulta simpático.

Seguimos encontrando, sin embargo, casos en los que la nueva acepción incluida por el Moliner-98 no estaba ni está en el diccionario de la Academia; es el caso de armario

armario 2 (Par.) Mueble donde se colocan libros.

h) Ya hemos vistos muchas acepciones (abarajar, apochongarse, aparceria, amansador) que afectan también al grupo de nuevas acepciones de Uruguay. Destacamos en este grupo sólo aquellas que en el $D R A E-2001$ ya no llevan la marca de Uruguay, como es el caso de abriboca, agauchado, ;alalá! y ampalaba o ampalagua 
abriboca 1 (Arg., Ur.) adj. y n. Distraído: que no se da cuenta de lo que pasa a su alrededor o no pone atención en lo que hace.

agauchado, -a (Arg., Chi., Par., Ur.) Participio adjetivo de "agauchar[se]". Parecido a los gauchos o a sus cosas.

¡alalá! (Guat., Ur.) interj. Expresa asombro o admiración.

ampalaba o ampalagua (Arg., Chi., Par., Ur.) f. Nombre de varias serpientes americanas de gran tamaño.

i) Entre las acepciones con marca de Venezuela ya hemos visto el caso de alfombra y arrochelarse, que no aparecen ni aparecían en el diccionario de la Academia; en las mismas circunstancias está la acepción de alfiler

alfiler 4 (Cuba, Pan., P. Rico, R. Dom., Ven.) *Imperdible.

Destaca también la cantidad de acepciones que, según el nuevo diccionario académico, ya no deben llevar la marca de Venezuela; es el caso de abacora, abacorar, aguado, amalayar, amasandero, ancheta y agujeta

abacora (Antill., Ven.) f. Albacora (pez).

abacorar 1 (Antill., Ven.) tr. Acosar, *perseguir.

aguado 4 (C. Rica, Guat., Méj., Pan., Ven.) adj. *Débil, flojo.

amalayar 2 (Am. C., Col., Méj., Ven.) tr. Desear vivamente algo.

amasandero, -a (Arg., Chi., Col., Ven.) n. Persona que amasa la harina para hacer pan.

ancheta 6 (Col., Ven.) Gratificación, propina.

agujeta 4 (And., Ven.) Alfiler largo que se colocan las mujeres en el sombrero para sujetarlo.

j) De las dos nuevas acepciones con marca de Río de la Plata sólo destaca la desaparición de la acepción de amuchar (ya vista) en el DRAE-2001.

El gráfico número 5 que ofrecemos a continuación agrupa los tres países que, siguiendo la estructuración jerarquizada de las marcas geográficas que ofrece el formato electrónico del nuevo Moliner, han de incluirse en la zona de Antillas (Cuba, Puerto Rico y República Dominicana).

R. Dom.

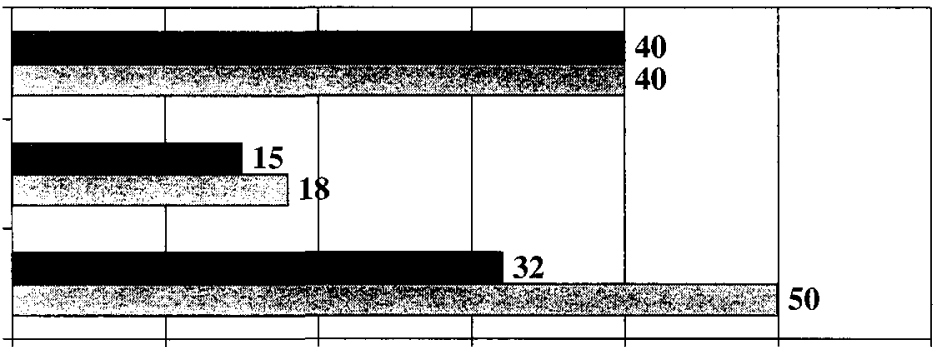

- Nuevas

P. Rico

Cuba

0

10

20

30

40

50

60

Gráfico 5. Países de Antillas. 
La lectura del gráfico es simple: de las 50 acepciones que aparecen en el Moliner- 98 con la marca de Cuba ( $\mathrm{Cuba}), 32$ han sido incluidas para esta edición; de las 18 acepciones con marca de Puerto Rico (P. Rico), 15 son nuevas; y, por último, las 40 acepciones que recoge con la marca de República Dominicana $(R$. Dom.) son nuevas acepciones para esta segunda edición del Moliner. Quiere esto decir que de un total de 108 acepciones recogidas, 87 son nuevas, más de un $80 \%$.

Encontramos muchas acepciones con alguna de estas tres marcas que se presentan tal y como estaban presentadas en el $D R A E-92$ y que así se mantienen, salvo alguna adición o supresión en el $D R A E-2001$. Destacaremos en primer lugar un grupo de acepciones que no han sido corregidas en el actual diccionario académico sino que han sido suprimidas; es el caso de aliblanca, amacharse, atagallar, acotejar (ya vista), de abulandro y aplazar
aliblanca 2 (Cuba) Cierta paloma salvaje. hembra.
atagallar 2 (Cuba, R. Dom.) Trabajar atosigado por alguna cosa. atagallar 3 (Cuba, R. Dom.) Anhelar.
de abulandro 1 ( $R$. Dom.) Aplicado al juego, sin arriesgar dinero. de abulandro 2 (R. Dom.) De mentirijillas. aplazar 3 (R. Dom.) prnl. recípr. Amancebarse.

amacharse 1 (Cuba, P. Rico, R. Dom.) prnl. Volverse estéril una planta o un animal

También encontramos casos que no parecen seguir lo que han hecho los diccionarios académicos; así ocurre en acaguasarse, incluida en el Moliner-98 sólo como de Cuba cuando el DRAE-92 ya la marcaba también de Granada; o en ajustador, que no viene en el $D R A E-92$, y en el $D R A E-2001$ se ha incluido, pero no de Cuba sino general; tampoco la acepción de argolla venía en el DRAE-92, y el nuevo diccionario académico sigue en este caso al Moliner-98 y la incluye como de Cuba (y también de Venezuela)

acaguasarse (... Cuba) prnl. Crecer poco el tallo de la caña de azúcar y multiplicarse en cambio sus hojas.

ajustador 4 (Cuba) *Sostén (prenda femenina).

argolla 9 (Cuba) *Pendiente en forma de aro.

Seguimos encontrando, además, nuevas acepciones que no aparecen, y tampoco aparecían en el diccionario de la Academia; algunas de ellas ya las hemos visto en los grupos anteriores, como abrigo, alfiler, alfombra, alfombra limpiadora, otras son absorbente, hacer ambulancia, anaquel (no el ya visto), acordeón, alambre, aposento y arito

absorbente 4 (Cuba, R. Dom.) *Pajita: tubo delgado para sorber líquidos.

hacer ambulancia (Cuba). Hacer *autostop.

anaquel 3 (P. Rico) Mueble donde se colocan libros.

acordeón 3 (R. Dom.) *Armónica.

alambre 6 (R. Dom.) Tendedero: dispositivo de alambres, cuerdas, etc., donde se tiende la ropa.

aposento 2 (R. Dom.) Dormitorio.

arito (R. Dom.) m. *Pendiente en forma de aro. 
En el gráfico 6 que ofrecemos a continuación aparece el conjunto de las marcas "americanas" con el número de acepciones nuevas. Destacan Argentina y Colombia en las marcas concretas de países, y otro buen número supera la treintena de nuevas acepciones ${ }^{13}$. Se trata de una importante cantidad de nuevas acepciones, sobre todo en comparación con las escasas novedades que veíamos del español peninsular ${ }^{14}$.

\section{Acepciones nuevas con cada una de las marcas "americanas"}

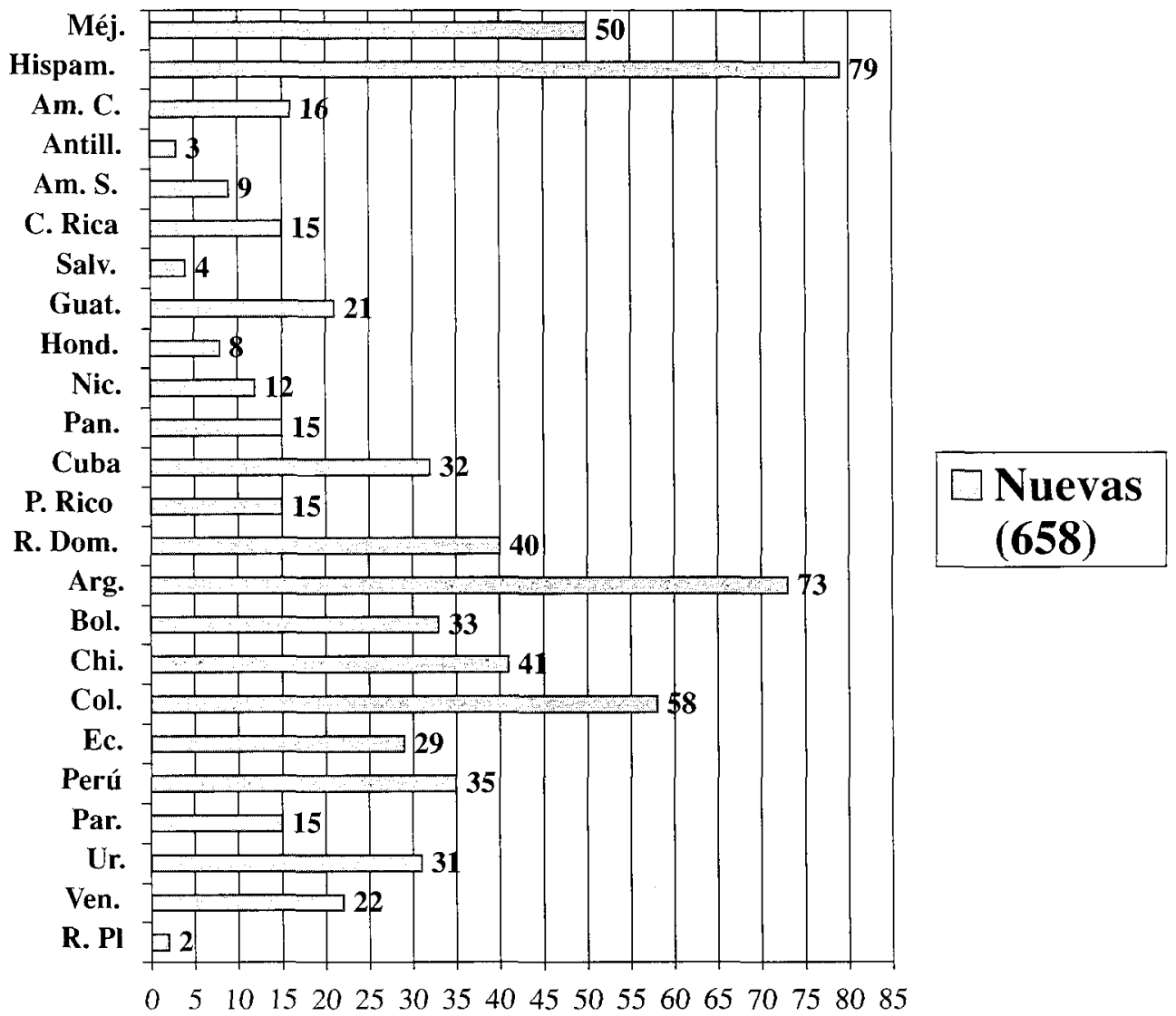

GRÁFICO 6

13 Recuérdese que estamos hablando de una sola letra del diccionario.

14 Como es sabido, el aumento de americanismos en la última edición académica ha sido muy importante; el propio Preámbulo del diccionario dice así: "se ha más que duplicado el número de americanismos en artículos, acepciones y marcas". Según los gráficos y estadísticas que sobre la nueva edición del diccionario aparecen en la página web de la RAE se ha pasado de 8.120 acepciones americanas a 18.749 , y de 12.494 marcas a 28.171 . Con respecto a los dialectalismos peninsulares la tendencia, sin embargo, parece la contraria: de 4.467 acepciones dialectales españolas se ha pasado a 3.772 , y de 5.397 marcas a 4.683 . 
Hemos podido comprobar, tras el análisis de este primer grupo del primer bloque, que la mayor parte de estas nuevas acepciones están en el diccionario académico. Sin embargo, muchas de ellas han sido modificadas, bien con la adición de otros países, bien con la supresión del país consultado, o bien con la eliminación de toda la acepción.

Podemos concluir, pues, aunque parcialmente ${ }^{15}$, que el diccionario si bien no es la fuente más idónea para un estudio teórico sobre la neología, sí refleja el cambio lingüístico que se produce diariamente en la sociedad. El diccionario, como el producto social más importante con el que cuenta una lengua, recoge dicho cambio. Es verdad que este primer grupo es el menos representativo, en comparación con los otros grupos que analizaremos. Es el grupo en el que menos innovaciones se han producido. Éstas se darán especialmente, como veremos en la segunda parte de nuestro trabajo, tanto en el grupo marcado técnicamente como en el bloque de "novedades sin marca".

\section{Referencias bibliográficas}

Azorín, D. (2000): “Formas de creación léxica en el lenguaje de los jóvenes a partir de un corpus oral del español contemporáneo", $L E A, \mathrm{XXU} / 2$, págs. 211-240.

Bastuji, J. (1974): “Aspects de la néologie sémantique", Langages, 36, págs. 6-19.

Cabré, T. (1993): La terminología. Teoría, metodologia, aplicaciones. Barcelona, Editorial Antártida / Empuries.

Casas Gómez, M. et alii (1998): Estudios sobre el Diccionario de uso del español de María Moliner. Cádiz, Universidad de Cádiz, Servicio de Publicaciones.

Dubois, J. et alii (1973): Diccionario de lingüistica. Madrid, Alianza Universidad.

Dubuc, R. (1999): Manual de terminologia. Trad. de Ileana Cabrera. Chile, RIL editores.

Fajardo, A. (1996-1997): "Las marcas lexicográficas: concepto y aplicación práctica en la Lexicografía española", Revista de Lexicografia, III, págs. 31-57.

Guerrero Ramos, G. (1997): Neologismos en el español actual. Madrid, Arco Libros.

Rey, A. (1976): "Le néologisme: un pseudo-concept?", Cahiers de Lexicologie, 28, págs. 3-7.

Mejri, S. (1995): La néologie lexicale. Tunis, Publications de la Faculté des Lettres de a Manouba.

Serie: Linguistique. Volume: IX.

Moliner, M.a (1966-1967): Diccionario de uso del español. 1.a ed. Madrid, Gredos. (Moliner-66). Moliner, M. ${ }^{a}$ (1998): Diccionario de uso del español. 2. ${ }^{a}$ ed. Madrid. Gredos. (Moliner-98). RAE (1992): Diccionario de la lengua española. 21. ${ }^{a}$ ed. Madrid, Espasa. (DRAE-92). RAE (2001): Diccionario de la lengua española. 22." ed. Madrid, Espasa. (DRAE-2001). Rondeau, G. (1984): Introduction à la terminologie. Québec, Gaëtan Morin. Seco, M. (1998): "Presentación". En Moliner, M. a (1998), págs. XI-XII.

15 Recuérdese que nos queda por analizar una parte importante de lo que hemos considerado renovación léxica en el diccionario de M. Moliner. 